\title{
New Method for Optimum Design of Pyramidal Horn Antennas
}

\author{
Leandro de Paula Santos Pereira, Marco Antonio Brasil Terada \\ Antenna Group, Electrical Engineering Dept., University of Brasilia - DF - terada@ unb.br
}

\begin{abstract}
This article introduces a procedure for determining the optimum design of pyramidal horn antennas. The efficiencies and phase errors in the optimum design are variables and depend on the design requirements. New equations are proposed for the optimum design, which can be solved numerically or analytically.
\end{abstract}

Index Terms - Antennas, Efficiency, Gain, Optimum Design, Pyramidal Horns.

\section{INTRODUCTION}

Pyramidal horn antennas are widely used in various applications in the microwave range due to its high gain, moderate bandwidth and low voltage standing wave ratio VSWR. Its construction is relativity simple and they are often used as feeders of reflectors and currently in applications where wide bandwidth is required, such as the technology WiMAX.

The pyramidal horn antenna design consists of determining their dimensions where the gain, the operation frequency and waveguide to which the antenna will be attached are given. Fig. 1 shows the antenna and their dimensions. A horn antenna design seeks to determine the values of $A, l_{H}$ or $R_{1}, l_{E}$ or $R_{2}$ and $B$, and these are related by [1]

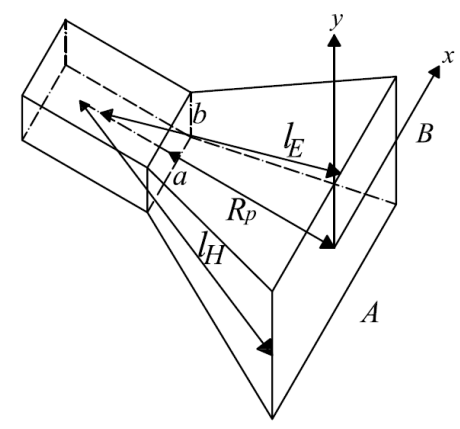

Fig. 1. Geometry of a pyramidal horn antenna.

$$
\begin{aligned}
& l_{H}^{2}=R_{1}^{2}+\left(\frac{A}{2}\right)^{2} \\
& l_{E}^{2}=R_{2}^{2}+\left(\frac{B}{2}\right)^{2}
\end{aligned}
$$

This work develops a new analytical design technique, using closed formulas based on equations that take into account the exact phase errors and optimize the gain relative to the $E$ - and $H$-planes. Thus, the procedure can be deemed as a consistent, closed form mathematical procedure, which can be solved numerically and/or analytically with high precision and accuracy. 
The usual methods do not consider the exact phase errors and, when they are used, it is assumed they are constants. Cozzens's method is based on empirical measurements which give results with a good accuracy [2], but they do not optimize the antenna gain in relation to the aperture dimensions. One advantage of the new technique is to provide less variation in the gain with respect to variations of the aperture dimensions, due to temperature variations for example, if certain conditions of the directivity curve are satisfied; e.g., if the curve is smooth, which is normally the case with practical antennas [1]. Thus, the designed antenna can yield a better stability in the gain for the frequency at which it was designed. Additionally, since the design is based on more accurate equations, the gain obtained from the design should be closer to reality.

\section{ON-AXIS GAIN OF PYRAMIDAL HORN}

There are several factors that influence the on-axis gain of pyramidal horns. When calculating the gain, it is usually assumed that only the dominant mode $\mathrm{TE}_{10}$ is present and propagating within the antenna; however this does not occur, because when the dimensions of the antenna become larger, other higher-modes will appear in the antenna aperture, changing its gain [3].

Also, wave reflections are not included in the formulas as it propagates inside the antenna. With the increase of the aperture, the wave suffers reflections, increasing the value of VSWR. If the transition from waveguide to free space is made smoothly, there will be fewer reflections and impedance matching will perform better, resulting in higher operating bandwidth, for example. This occurs when the flare or aperture angle of the antenna is small and the value of the length of the antenna is large.

Another factor that is not normally taken into account is the oscillation of the gain of pyramidal with frequency due to multiple diffractions on the aperture edges of the $E$-plane with further reflections on the wall of the antenna [4]. These oscillations become increasingly smaller with increasing of the frequency.

Thus, it can be seen how complex may be the process of determining the gain of the antenna. The pyramidal antenna gain, ignoring the phenomena discussed above can be given by [1]

$$
G=\frac{\pi}{32}\left(\frac{\lambda}{a} D_{E}\right)\left(\frac{\lambda}{b} D_{H}\right)=\frac{4 \pi}{\lambda^{2}} \varepsilon_{a p} A B
$$

with

$$
\begin{aligned}
& D_{E}=\frac{a}{\lambda} \frac{32}{\pi} \frac{B}{\lambda} R_{E} \\
& D_{H}=\frac{b}{\lambda} \frac{32}{\pi} \frac{A}{\lambda} R_{H}
\end{aligned}
$$

where $G$ is the desired on-axis gain, $D_{E}$ and $D_{H}$ are the gains of the $E$ - and $H$-planes sectoral horns, and $R_{E}$ and $R_{H}$ are, respectively, the phase error aperture efficiencies in the $E$ - and $H$-plane, and $\varepsilon_{a p}$ is the aperture efficiency of the antenna. They are given by [1]: 


$$
\begin{gathered}
R_{E}=\frac{C^{2}(q)+S^{2}(q)}{q^{2}} \\
R_{H}=\frac{\pi^{2}}{64 t}\left\{\left[C\left(p_{1}\right)-C\left(p_{2}\right)\right]^{2}+\left[S\left(p_{1}\right)-S\left(p_{2}\right)\right]^{2}\right\}
\end{gathered}
$$

where $C($ ) and $S($ ) functions are Fresnel integrals and

$$
\begin{gathered}
q=2 \sqrt{s} \\
p_{1}=2 \sqrt{t}\left(1+\frac{1}{8 t}\right) \\
p_{2}=2 \sqrt{t}\left(-1+\frac{1}{8 t}\right)
\end{gathered}
$$

The values of $q, p_{1}$ and $p_{2}$ are dependent on the values of phase errors $t$ in the $H$-plane and $s$ in the E-plane, given by

$$
\begin{gathered}
t=\frac{A^{2}}{8 \lambda R_{1}} \\
s=\frac{B^{2}}{8 \lambda R_{2}}
\end{gathered}
$$

Equations (3) to (12) were derived by Schelkunoff [5]. These expressions use the geometrical optics theory and single diffraction at the edges of the aperture antenna. They are used to calculate the onaxis gain of pyramidal horns and the $H$-plane sectoral horns [6]. For $E$-plane sectoral horns, Jull's formula for calculating the gain is more accurate [7]. In addition, the maximum possible error calculated for Schelkunoff's gain formula using (3) is about $\pm 0,3 \mathrm{~dB}$ [8],[9].

The values of exact phase errors [10] result in a gain close to the measured values, as shown by Fig. 2. They are given by

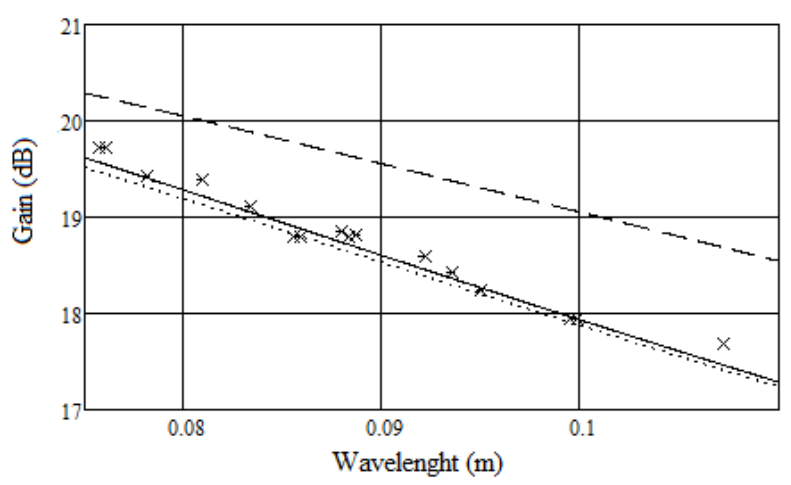

Fig. 2. Gain curves obtained for a WR284 waveguide with $A=32.41 \mathrm{~cm}, B=24 \mathrm{~cm}, l_{E}=42.15 \mathrm{~cm}$ and $l_{H}=47.45 \mathrm{~cm}$. ---analysis using exact phase errors, $\cdots$... analysis using quadratic phase errors, --- analysis using quadratic phase error in $H$-plane and Jull's formula in the $E$-plane [7], $\times \times \times$ measured values [4],[8].

$$
t_{e}=\left(\frac{A}{\lambda}\right)^{2} \frac{1}{8 t}\left\{\left[1+\left(\frac{\lambda}{A}\right)^{2} 16 t^{2}-1\right]^{1 / 2}-1\right\}
$$




$$
s_{e}=\left(\frac{B}{\lambda}\right)^{2} \frac{1}{8 s}\left\{\left[1+\left(\frac{\lambda}{B}\right)^{2} 16 s^{2}-1\right]^{1 / 2}-1\right\}
$$

Consequently, to obtain more accurate designs, it is necessary to use (13) and (14) instead of (11) and (12) in (8) to (10) to calculate the gain and analyzing the results, especially when the antenna length is small [10]. Jull's formula [7] gives more accurate result when is only used for E-plane sectoral antennas, because when it is used in the pyramidal antennas, the results are far from the measured, shown in Fig. 2. The effects of the fringe-currents at the edges on the axis-gain are different for pyramidal and $E$-plane sectoral horns, because the last have closely spaced edges whereas the former have widely spaced edges, which make these effects less significant on the gain of pyramidal antenna [7],[11].

Usually it is assumed that the optimum aperture efficiency $\varepsilon_{a p}$ in the design is 0.5 or 0.51 [1]. However, this value does not represent the optimum aperture efficiency, but is close to it. In this work, the optimal design is the result of the choice of the value of $A$ that maximizes the gain in $\mathrm{H}$ plane for a given axial length $R_{1}$, and also by choosing the value of $B$ that maximizes the gain in the $E$-plane for a given value of $R_{2}$. It is important to notice that the design is optimum only for the desired frequency of operation, and does not necessarily also maximizes the aperture efficiency due to the fact that the gain curve is smooth around its peak.

Commonly the quadratic phase errors are used with $t=0.375$ for the $H$-plane and $s=0.25$ for the $E$ plane [1]. These values do not optimize the gain or directivity, since these are derived from approximations of the aperture dimensions that maximize the gain for a given axial length. This approach lead to

$$
\begin{aligned}
& A \cong \sqrt{3 \lambda R_{1}} \\
& B \cong \sqrt{2 \lambda R_{2}}
\end{aligned}
$$

It is also possible to define the optimum aperture efficiency as 0.49 and optimum phase errors $t=$ 0.4 and $s=0.26[12]$.

\section{DEVELOPMENT OF AN OPTIMUM DESIGN PROCEDURE WITH A NUMERICAL AND AN ANALYTICAL SOLUTIONS}

There are many possibilities to design an antenna, which give several different results in the dimensions, but produce the same result in the gain. To find a single solution of the design, we have to obtain a minimum of equations relating the four dimensions that we want to achieve, given the value of the on-axis gain, the operating frequency and waveguide. The developed procedure uses (13) and (14) in (3) to (10), and for the antenna to be realizable, the following constrain has to be enforced:

$$
R_{1} \frac{A-a}{A}=R_{2} \frac{B-b}{B}
$$

For the optimum design, it is chosen $A$ for a fixed value of $R_{1}$ that maximizes the gain curve in the $H$-plane. This is done by deriving the expression of the gain or directivity (5) with respect to the 
dimension $A$ and, through a numerical solution, it is determined the root of this expression, i.e., it is determined $A$ in function of the independent value $R_{1}$ :

$$
\begin{aligned}
& f_{1}\left(A, R_{1}\right)=\frac{\partial D_{H}}{\partial A} \\
& A\left(R_{1}\right)=\operatorname{root}\left(f_{1}\right)
\end{aligned}
$$

where root ( ) is a numerical function that calculates the zeroes of its argument.

The same have to be done to the $E$-plane, where $B$ is obtained according to the value of $R_{2}$ :

$$
\begin{aligned}
& f_{2}\left(B, R_{2}\right)=\frac{\partial D_{E}}{\partial B} \\
& B\left(R_{2}\right)=\operatorname{root}\left(f_{2}\right)
\end{aligned}
$$

However, these expressions do not have a single root, but many, as can be seen in Fig. 3. Its curve of directivity has several points where its derivative is zero. This also happens with the directivity of the $H$-plane, although the oscillations of the curve are less intense. To select the value of $A$ and $B$ that maximizes the directivity curve, the root can be chosen so that it is within the range [13]:

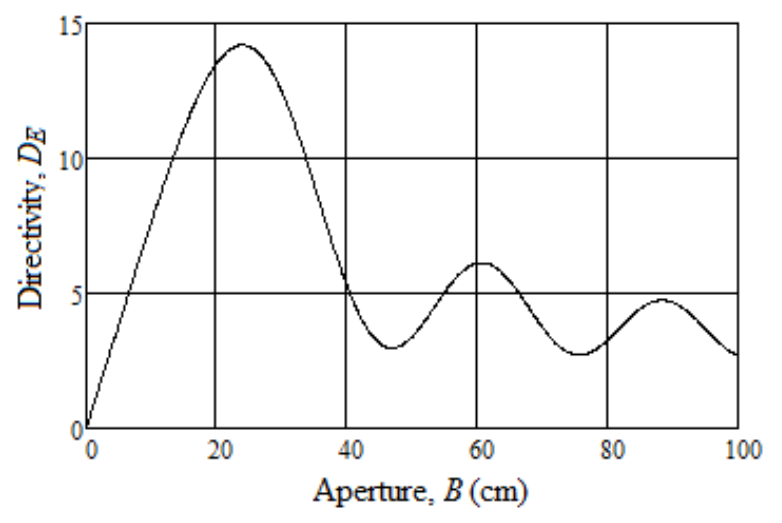

Fig. 3. Directivity curve computed with respect to the aperture $B$ with $R_{2}=23.65 \mathrm{~cm}$ for an operating frequency of $2.5 \mathrm{GHz}$ using a WR430 waveguide.

$$
\lambda\left(\frac{G}{2 \pi}\right)^{\frac{1}{2}}<A<\lambda\left(\frac{G}{\pi}\right)^{\frac{1}{2}}
$$

where $G$ is the pyramidal antenna gain. The range of $B$ can be found by the limits of $A$ when using

$$
B=\frac{\lambda^{2} G}{4 \pi \varepsilon_{a p} A}
$$

We can estimate $\varepsilon_{a p}=0.49$ to find the range of root $B$, although it is possible to use other values close to this.

After that, equation (21) is used in order for the dimensions of the antenna to be realizable. As $A$ is a function of $R_{1}$ and $B$ is a function of $R_{2}$, an equation is obtained with two independent variables, $R_{1}$ and $R_{2}$. Thus, $R_{1}$ can be found as the root that satisfies (16) for a given value of $R_{2}$ :

$$
\begin{gathered}
f_{3}\left(R_{1}, R_{2}\right)=R_{1} \frac{A-a}{A}-R_{2} \frac{B-b}{B} \\
R_{1}\left(R_{2}\right)=\operatorname{root}\left(f_{3}\right)
\end{gathered}
$$


Using the previous result in (3), with $G$ being the desired gain value, the numerical solution of the design is found through the $R_{2}$ value, since all other dimensions are dependent on $R_{2}$ :

$$
\begin{gathered}
f_{4}\left(R_{2}\right)=G-\frac{\pi}{32}\left(\frac{\lambda}{a} D_{E}\right)\left(\frac{\lambda}{b} D_{H}\right) \\
R_{2}=\operatorname{root}\left(f_{4}\right)
\end{gathered}
$$

It is possible to develop an analytical method for this design based on the results, where it is verified that the exact phase errors vary with the requirements of gain, the dimensions of the waveguide and the operation frequency. When a waveguide with $2 \leq a / b \leq 2.5$ and frequency in the range $1<\lambda / a<1.7$ are used, the changes in optimal $t_{e}$ and $s_{e}$ become negligible with the variations of $a, b$ and $\lambda$. The commercial waveguides for standard pyramidal antennas have dimensions in theses ranges, which are also used in Cozzens's method [2]. The exact phase errors are dependent on the desired gain in accordance with the following approaches:

$$
\begin{aligned}
& t_{e} \cong 0,3967+\frac{0,6281}{G} \\
& s_{e} \cong 0,262+\frac{0,3178}{G}
\end{aligned}
$$

where $G$ is given in absolute value. These equations can be used for standard antennas with gains between 10 and $25 \mathrm{~dB}$. It can be seen that if the desired gain is high, $t_{e}$ and $s_{e}$ approach 0.3967 and 0.262 , respectively. When using (11) and (13), one arrives at

$$
A=2 \sqrt{t_{e} \lambda\left(t_{e} \lambda+2 R_{1}\right)}
$$

Similarly, with (12) and (14)

$$
B=2 \sqrt{s_{e} \lambda\left(s_{e} \lambda+2 R_{2}\right)}
$$

Isolating $A$ in (3) and substituting in (25), the following equation is obtained

$$
R_{1}=\frac{G^{2} \lambda^{4}}{16 \pi^{2} \varepsilon_{a p}^{2} B^{2} 8 \lambda t_{e}}-\frac{t_{e} \lambda}{2}
$$

Isolating $R_{2}$ in (26) and substituting it together with (27) and (3) in (17), one arrives at

$$
\frac{B^{4}}{8 \lambda s_{e}}-\frac{1}{\lambda}\left(\frac{b}{8 s_{e}}+\frac{2 \pi a \varepsilon_{a p} t_{e}}{G}\right) B^{3}+\frac{\lambda}{2}\left(t_{e}-s_{e}\right) B^{2}+\lambda\left(\frac{s_{e} b}{2}+\frac{a G}{32 \pi \varepsilon_{a p} t_{e}}\right) B-\frac{G^{2} \lambda^{3}}{128 \pi^{2} \varepsilon_{a p}{ }^{2} t_{e}}=0
$$

The value of $\varepsilon_{a p}$ is exclusively determined by the use of (23) and (24). Equation (28) can be solved numerically or analytically. Hence, the approximated optimal design can be calculated using (28) with (23) and (24).

\section{RESULTS}

Three designs using three different techniques for each were made, where all were calculated numerically in order to compare the results of optimum designs. Thus, the results obtained with the procedure herein introduced (optimum design) were compared to the Cozzens's method [2] and with the traditional design with $t=0.375 s=0.25$. 
Normally, in traditional designs, the exact phase errors are not used in the equations of directivity for the $H$-and $E$-planes, but these were used in this work.

Cozzens's method is a design based on empirical measurements, which produces accurate results [2]. As the results are based on measurements, its design already includes all electromagnetic effects that can affect the gain. Tables I, II and III present the three designs respectively. In these three designs, it was sought to design short, long and intermediate antennas to examine different situations that may arise in practice.

TABLE I. RESULTS OF THE DESIGNS OF A PYRAMIDAL HORN ANTENNA WITH GAIN OF 18 dB USING A WR137 WAVEGUIDE AND FREQUENCY OF $6 \mathrm{GHZ}(\lambda=5 \mathrm{CM})$

\begin{tabular}{cccc}
\hline \multirow{2}{*}{ Dimensions } & \multicolumn{3}{c}{ Designs (Intermediate $\boldsymbol{R}_{\boldsymbol{p}}$ ) } \\
\cline { 2 - 4 } & Optimum & Cozzens & Traditional \\
\hline$R_{2}(\mathrm{~cm})$ & 18.20 & 17.47 & 18.35 \\
$R_{1}(\mathrm{~cm})$ & 19.94 & 19.04 & 20.26 \\
$B(\mathrm{~cm})$ & 14.19 & 13.75 & 13.54 \\
$A(\mathrm{~cm})$ & 18.46 & 18.57 & 17.43 \\
$R_{p}(\mathrm{~cm})$ & 16.17 & 15.46 & 16.21 \\
\hline
\end{tabular}

In Cozzens's method, in comparison with the designs developed in this work, the value of $R_{p}$ is always smaller for short, intermediate and long antennas, whereas the traditional design always results in longer $R_{p}$. Thus, the optimum design results in an intermediate value of axial length of the antenna, which is closest to the traditional design.

TABLE II. RESULTS OF THE DESIGNS OF A PYRAMIDAL HORN ANTENNA WITH GAIN OF 23 dB USING A WR62 WAVEGUIDE AND FREQUENCY OF $14 \mathrm{GHZ}(\lambda=2.14 \mathrm{CM})$

\begin{tabular}{cccc}
\hline \multirow{2}{*}{ Dimensions } & \multicolumn{3}{c}{ Designs (Large $\boldsymbol{R}_{\boldsymbol{p}}$ ) } \\
\cline { 2 - 4 } & Optimum & Cozzens & Traditional \\
\hline$R_{2}(\mathrm{~cm})$ & 25.97 & 26.00 & 26.06 \\
$R_{1}(\mathrm{~cm})$ & 27.21 & 27.06 & 27.37 \\
$B(\mathrm{~cm})$ & 10.90 & 10.48 & 10.57 \\
$A(\mathrm{~cm})$ & 13.76 & 14.15 & 13.27 \\
$R_{p}(\mathrm{~cm})$ & 24.08 & 24.04 & 24.11 \\
\hline
\end{tabular}

Moreover, the values of $R_{1}$ and $R_{2}$ in the optimum design are always lower than in the traditional design, due to the maximization of gain relative to the aperture size. In general, these dimensions are intermediate values between Cozzens's method and traditional design.

TABLE III. RESULTS OF THE DESIGNS OF A PYRAMIDAL HORN ANTENNA WITH GAIN OF 14 dB USING A WR430 WAVEGUIDE AND FREQUENCY OF $2 \mathrm{GHZ}(\lambda=15 \mathrm{CM})$

\begin{tabular}{cccc}
\hline \multirow{2}{*}{ Dimensions } & \multicolumn{3}{c}{ Designs $\left(\right.$ Small $\boldsymbol{R}_{\boldsymbol{p}}$ ) } \\
\cline { 2 - 4 } & Optimum & Cozzens & Traditional \\
\hline$R_{2}(\mathrm{~cm})$ & 19.87 & 17.13 & 20.41 \\
$R_{1}(\mathrm{~cm})$ & 22.67 & 19.64 & 23.86 \\
$B(\mathrm{~cm})$ & 26.85 & 26.03 & 24.74 \\
$A(\mathrm{~cm})$ & 36.19 & 35.15 & 32.77 \\
$R_{p}(\mathrm{~cm})$ & 15.83 & 13.54 & 15.91 \\
\hline
\end{tabular}

With respect to the aperture $B$, the optimum design results in larger dimensions than the other two, 
approaching more the results obtained with Cozzens's method. For the dimension $A$, in general, the optimum design results in an intermediate value of the other two procedures, except for short antennas, where its value is greater than the others, as shown in Table III. In addition, its value is closer to Cozzens' method.

The dimensions obtained by the optimum design herein presented are values that lie in the range of Cozzens's method and the traditional design, whose aperture dimensions are closer to Cozzens's method and axial length dimensions closer to the traditional design.

In order to compare the new optimum design with a procedure more recently available in the literature, three news designs were performed and compared with a method using an improved set of formulas combined with Particle Swarm Optimization [14]. Tables IV, V e VI show the results.

TABLE IV. RESULTS OF THE DESIGNS OF A PYRAMIDAL HORN ANTENNA WITH GAIN OF 21.75 dB USING A WR137 WAVEGUIDE AND FREQUENCY OF $6.779 \mathrm{GHZ}(\lambda=4.43 \mathrm{CM})$

\begin{tabular}{ccc}
\hline \multirow{2}{*}{ Dimensions } & \multicolumn{2}{c}{ Designs (Intermediate $\boldsymbol{R}_{\boldsymbol{p}}$ ) } \\
\cline { 2 - 3 } & Optimum & Method in [14] \\
\hline$A(\mathrm{~cm})$ & 24.78 & 24.50 \\
$B(\mathrm{~cm})$ & 19.40 & 19.27 \\
\hline
\end{tabular}

TABLE V. RESULTS OF THE DESIGNS OF A PYRAMIDAL HORN ANTENNA WITH GAIN OF 23.5 dB USING A WR62 WAVEGUIDE AND FREQUENCY OF $14.95 \mathrm{GHZ}(\lambda=2.007 \mathrm{CM})$

\begin{tabular}{ccc}
\hline \multirow{2}{*}{ Dimensions } & \multicolumn{2}{c}{ Designs $\left(\right.$ Large $\left.\boldsymbol{R}_{\boldsymbol{p}}\right)$} \\
\cline { 2 - 3 } & Optimum & Method in [14] \\
\hline$A(\mathrm{~cm})$ & 13.65 & 13.55 \\
$B(\mathrm{~cm})$ & 10.80 & 10.76 \\
\hline
\end{tabular}

TABLE VI. RESULTS OF THE DESIGNS OF A PYRAMIDAL HORN ANTENNA WITH GAIN OF 16.5 dB USING A WR430 WAVEGUIDE AND FREQUENCY OF $2.163 \mathrm{GHZ}(\lambda=13.87 \mathrm{CM})$

\begin{tabular}{ccc}
\hline \multirow{2}{*}{ Dimensions } & \multicolumn{2}{c}{ Designs $\left(\right.$ Small $\left.\boldsymbol{R}_{\boldsymbol{p}}\right)$} \\
\cline { 2 - 3 } & Optimum & Method in [14] \\
\hline$A(\mathrm{~cm})$ & 43.57 & 41.90 \\
$B(\mathrm{~cm})$ & 33.07 & 32.16 \\
\hline
\end{tabular}

Accordingly to the results of Tables IV, V and VI, the $A$ and $B$ apertures for the proposed method is larger than the method in [14]. In all designs, the results are close. However, there are more differences in the values in the design of short antennas than with the design of long antennas, as seen by the Tables V and VI. This occurs because the method in [14] uses constant phase errors, unlike the proposed method, where these are variables to guarantee that the exact phase errors are accounted.

One of the advantages in using the optimum design is the possibility of the gain to be more stable with the aperture dimensions, if certain conditions on the directivity curve are satisfied, like the smoothness of the curve. For the values of $R_{1}$ and $R_{2}$, if the values of $A$ and $B$ are increased or decreased, the gain is reduced, meaning that the pyramidal antenna gain is in its optimum point, where its derivative is zero.

In addition, analyses were done for all three techniques obtained for each design requirement. Tables VII, VIII and IX present the results of the analyses, where we used (3) to (10) with (13) and 
(14) in the equations of directivity.

\begin{tabular}{|c|c|c|c|}
\hline \multirow{2}{*}{ Parameters } & \multicolumn{3}{|c|}{ Designs } \\
\hline & Optimum & Cozzens & Traditional \\
\hline$s$ & 0.277 & 0.271 & 0.250 \\
\hline$s_{e}$ & 0.267 & 0.261 & 0.242 \\
\hline$t$ & 0.428 & 0453 & 0.375 \\
\hline$t_{e}$ & 0.401 & 0.429 & 0.359 \\
\hline$\varepsilon_{a p}$ & 0.479 & 0.471 & 0.532 \\
\hline$G(\mathrm{~dB})$ & 18.00 & 17.81 & 18.00 \\
\hline$\partial D_{E} / \partial B\left(\mathrm{~m}^{-1}\right)$ & 0 & 5.10 & 20.73 \\
\hline$\partial D_{H} / \partial A\left(\mathrm{~m}^{-1}\right)$ & 0 & -4.53 & 10.55 \\
\hline
\end{tabular}

TABLE VIII. ANALYSIS OF RESULTS OF THE DESIGNS OF TABLE II

\begin{tabular}{cccc}
\hline \multirow{2}{*}{ Parameters } & \multicolumn{3}{c}{ Designs } \\
\cline { 2 - 4 } & Optimum & Cozzens & Traditional \\
\hline$s$ & 0.267 & 0.246 & 0.250 \\
$s_{e}$ & 0.264 & 0.244 & 0.247 \\
$t$ & 0.406 & 0.432 & 0.375 \\
$t_{e}$ & 0.400 & 0.424 & 0.370 \\
$\varepsilon_{a p}$ & 0.486 & 0.488 & 0.520 \\
$G(\mathrm{~dB})$ & 23.00 & 22.97 & 23.00 \\
$\partial D_{E} / \partial B\left(\mathrm{~m}^{-1}\right)$ & 0 & 41.84 & 34.61 \\
$\partial D_{H} / \partial A\left(\mathrm{~m}^{-1}\right)$ & 0 & -14.97 & 18.85 \\
\hline
\end{tabular}

TABLE IX. ANALYSIS OF RESULTS OF THE DESIGNS OF TABLE III

\begin{tabular}{cccc}
\hline \multirow{2}{*}{ Parameters } & \multicolumn{3}{c}{ Designs } \\
\cline { 2 - 4 } & Optimum & Cozzens & Traditional \\
\hline$s$ & 0.302 & 0.330 & 0.250 \\
$s_{e}$ & 0.274 & 0.292 & 0.230 \\
$t$ & 0.481 & 0.524 & 0.375 \\
$t_{e}$ & 0.422 & 0.448 & 0.339 \\
$\varepsilon_{a p}$ & 0.463 & 0.430 & 0.555 \\
$G(\mathrm{~dB})$ & 14.00 & 13.43 & 14.00 \\
$\partial D_{E} / \partial B\left(\mathrm{~m}^{-1}\right)$ & 0 & -4.47 & 11.87 \\
$\partial D_{H} / \partial A\left(\mathrm{~m}^{-1}\right)$ & 0 & -1.57 & 6.55 \\
\hline
\end{tabular}

For Tables VII, VIII and IX, the values of exact phase errors are variables in the traditional design, including the aperture efficiency, because parameters of correction of phase errors were used in the analysis of the gain formula. However the phase errors although treated as variables are not adjusted to be the exact ones, as in the new proposed procedure.

In the optimum design, the quadratic phase errors are also variables. In Cozzens's method, the efficiencies and phase errors are also variables, determined by the procedure.

If (11) and (12) were used in the gain equation and in analysis of the optimum design, the values of phase errors and the aperture efficiency would be constant, and approximated by $t=0.4, t_{e}=0.39, s=$ $0.26, s_{e}=0.26$ and $\varepsilon_{a p}=0.49$, independent of the antenna to be long or short. But when using quadratic phase errors in the formulas of directivity, the results are less accurate, especially for short 
antennas. In addition, as the gain calculated by (11) and (12) are smaller than when using exact phase errors, the designed antenna results in higher dimensions.

In Table VIII, the values of $t$ and $t_{e}$ are approximately equal, with the value of 0.4 . The same is true for the values of $s$ and $s_{e}$, approaching 0.26 . The efficiency $\varepsilon_{a p}$ is close to 0.49 . However, in the optimum design, their values depend mainly on the gain requirements. For long antennas, i.e., $R_{1}>>$ $A / 2$ e $R_{2} \gg B / 2$, the exact phase errors and quadratic tend to equalize as well as aperture efficiency tend to 0.49 . Such situations arise when designing an antenna for high gain.

For Table IX, it is seen that the exact and quadratic phase errors differ considerably. When the design requirements are for low gain antennas, the procedure results in short antennas, with flare angles that are large, and the exact and quadratic phase errors diverge with the aperture efficiency being dramatically reduced. Fig. 4 shows the variation of $\varepsilon_{a p}$ with the desired gain and Fig. 5 shows the variation of $t_{e}$ and $t$ to the desired gain.

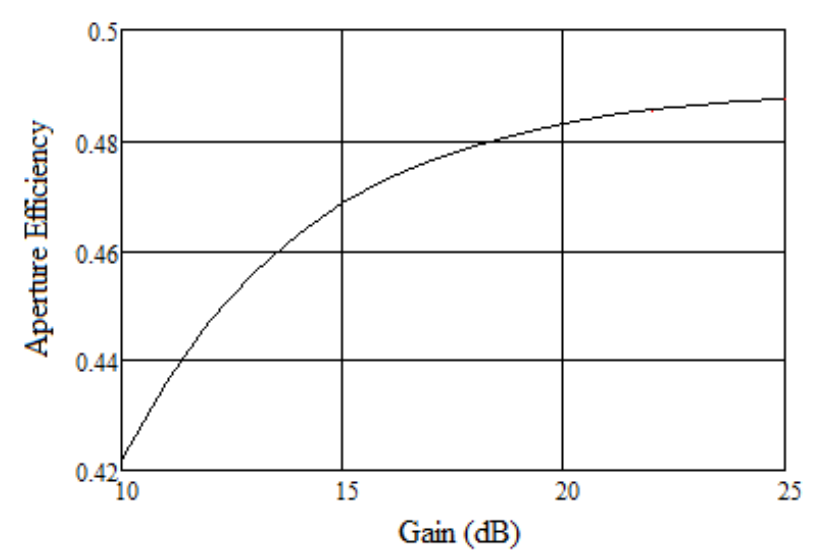

Fig. 4. Variation of the aperture efficiency obtained from the optimum design with a WR430 waveguide and a frequency of $2.5 \mathrm{GHz}$.

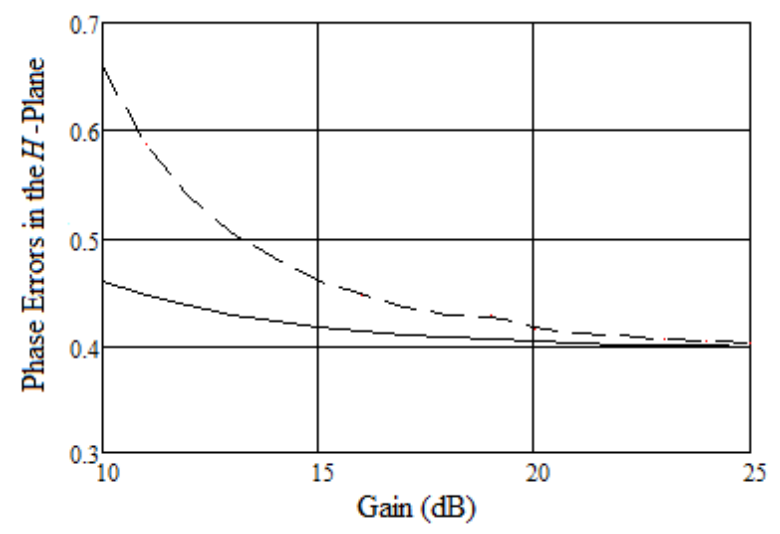

Fig. 5 Variation of the phase errors in the $H$-plane with respect to the desired gain obtained from the optimum design with a WR430 waveguide and a frequency of $2.5 \mathrm{GHz}$. --- Exact phase error, - - - Quadratic phase error.

In the all designs, Cozzens's method resulted in negatives values of the derivatives of the gain in the $H$-plane. In Table IX, the derivative of the gain in the $E$-plane is also negative. This means that the designed apertures passed the optimum point of design in the curve of directivity.

Similarly, it can be achieved the optimum design using (23) to (28) instead of (17) to (22). With 
these equations, the same designs in Tables I, II and III were developed, whose results are shown in Tables X, XI and XII. The analyses of these designs use (3) to (10) with (13) and (14).

TABLE X. RESUlTS OF THE APPROXIMATED OPTIMUM DESIGN OF A PYRAMIDAL HORN ANTENNA WITH A GAIN OF 18 dB USING A WR137 WAVEGUIDE AND A FREQUENCY OF 6 GHZ $(\lambda=5 \mathrm{CM})$

\begin{tabular}{cccc}
\hline \multicolumn{4}{c}{ Approximated Optimum Design } \\
\hline Design Dimensions & \multicolumn{2}{c}{ Analyzed Parameters } \\
\hline$R_{2}(\mathrm{~cm})$ & 18.20 & $s_{e}$ & 0.267 \\
$R_{1}(\mathrm{~cm})$ & 19.94 & $t_{e}$ & 0.401 \\
$B(\mathrm{~cm})$ & 14.20 & $\mathcal{E}_{a p}$ & 0.479 \\
$A(\mathrm{~cm})$ & 18.46 & $G(\mathrm{~dB})$ & 18 \\
$R_{p}(\mathrm{~cm})$ & 16.17 & & \\
\hline
\end{tabular}

TABLE XI. RESULTS OF THE APPROXIMATED OPTIMUM DESIGN OF A PYRAMIDAL HORN ANTENNA WITH A GAIN OF 23 dB USING A WR62 WAVEGUIDE AND A FREQUENCY OF $14 \mathrm{GHZ}(\lambda=2.14 \mathrm{CM})$

\begin{tabular}{cccc}
\hline \multicolumn{4}{c}{ Approximated Optimum Design } \\
\hline \multicolumn{3}{c}{ Design Dimensions } & \multicolumn{3}{c}{ Analyzed Parameters } \\
\hline$R_{2}(\mathrm{~cm})$ & 25.97 & $s_{e}$ & 0.264 \\
$R_{1}(\mathrm{~cm})$ & 27.21 & $t_{e}$ & 0.400 \\
$B(\mathrm{~cm})$ & 10.89 & $\varepsilon_{a p}$ & 0.486 \\
$A(\mathrm{~cm})$ & 13.76 & $G(\mathrm{~dB})$ & 23 \\
$R_{p}(\mathrm{~cm})$ & 24.08 & & \\
\hline
\end{tabular}

TABLE XII. RESULTS OF THE APPROXIMATED OPTIMUM DESIGN OF A PYRAMIDAL HORN ANTENNA WITH A GAIN OF 14 dB USING A WR430 WAVEGUIDE AND A FREQUENCY OF $2 \mathrm{GHZ}(\lambda=15 \mathrm{CM})$

\begin{tabular}{cccc}
\hline \multicolumn{4}{c}{ Approximated Optimum Design } \\
\hline Design Dimensions & \multicolumn{3}{c}{ Analyzed Parameters } \\
\hline$R_{2}(\mathrm{~cm})$ & 19.87 & $s_{e}$ & 0.275 \\
$R_{1}(\mathrm{~cm})$ & 22.68 & $t_{e}$ & 0.422 \\
$B(\mathrm{~cm})$ & 26.88 & $\varepsilon_{a p}$ & 0.463 \\
$A(\mathrm{~cm})$ & 36.16 & $G(\mathrm{~dB})$ & 14 \\
$R_{p}(\mathrm{~cm})$ & 15.83 & & \\
\hline
\end{tabular}

Verifying the results in Tables X, XI and XII, the results are the same as calculated numerically by (17) to (22), except for a small difference in the $B$ value for all cases, and in the $R_{1}$ and $A$ values for short antennas. The values of $t_{e}$ and $s_{e}$ obtained using (23) and (24) are close to the results shown in Tables VII, VIII and IX. Therefore, for the optimum design, these news proposed equations can be used to design a pyramidal horn antenna.

\section{CONCLUSIONS}

Usually, design procedures for pyramidal horn antennas employ constant values for the quadratic phase errors. This study showed that these values are not constant when using relations (18) and (19) with the exact phase errors and equations (25) and (26). The variations depend on the desired gain of the antenna. For long antennas, it can be assumed that the aperture efficiency is close to 0.49. For short antennas, the efficiency can reach values as low as 0.42 . It is also shown that the optimum design proposed results in aperture dimensions larger than the method in [14], and in intermediate dimensions between Cozzens's method and the traditional design $(t=0.375, s=0.25)$. 


\section{REFERENCES}

[1] W. L. Stutzman, and G. A. Thiele, Antenna Theory and Design, 2nd ed., John Wiley and Sons, 1998, pp. 300-314.

[2] D. E Cozzens, "Tables Ease Horn Design," in Microwaves, pp.37 -39, March 1966.

[3] K. Liu, C. A. Balanis, C. R. Birtcher and G. C. Barber, "Analysis of Pyramidal Horn Antennas Using Moment Method", in IEEE Trans Antennas Propagat., vol. AP-41, pp. 1379-1389, October 1993.

[4] E. V. Jull, "Errors in the Predicted Gain of Pyramidal Horns" in IEEE Trans Antennas Propagat., vol. AP-21, pp. 2531, January 1973.

[5] S. A. Schelkunoff, Electromagnetic Waves. New York: Van Nostrand Rheinhold, 1943, pp. 360-364.

[6] K. T. Selvan, "An Approximate Generalization of Schelkunoff's Horn-Gain Formulas", in IEEE Trans Antennas Propagat., vol. 47, pp. 1001-1004, June 1999.

[7] E. V. Jull, and L. E. Allan, "Gain of a E-Plane Sectoral Horn - A Failure of the Kirchhoff Theory and a New Proposal" in IEEE Trans Antennas Propagat., vol. AP-22, pp. 221-226, March 1974.

[8] W. T. Slayton, "Design and Calibration of Microwave Antenna Gain Standards" U.S. Naval Res. Lab., Washington, D.C., Rep. 4433, November 1954.

[9] J. L. Teo, K. T. Selvan, "On the Optimum Pyramidal-Horn Design Methods", in Int J RF and Microwave CAE 16, pp.561-564, September 2006.

[10] M. J. Maybell, and P. S. Simon, "Pyramidal Horn Gain Calculation with Improved Accuracy", in IEEE Trans Antennas Propagat., vol. AP-41, pp. 884-889, July 1993.

[11] K. T. Selvan, R. Sivaramakrishnan, K. R. Kini, and D. R. Poddar, "Experimental Verification of the Generalized Schelkunoff's Horn-Gain Formulas for Sectoral Horns", in IEEE Trans Antennas Propagat., vol. 50, pp. 875-877, June 2002.

[12] T. A. Milligan, Modern Antenna Design, 2nd ed., John Wiley and Sons, 2005, pp. 343.

[13] J. F Aurand, in "A New Design Procedure for Optimum Gain Pyramidal Horns", in Antennas and Propagation Society International Symposium, AP-S Digest, 1988.

[14] Y. Najjar, M. Moneer, N. Dib, "Design of Optimum Gain Pyramidal Horn with Improved Formulas Using Particle Swarm Optimization", in Int J RF and Microwave CAE 17, pp.505-511, June 2007. 